\title{
Using matrix stability for variable telegraph partial differential equation
}

\author{
Mahmut Modanli ${ }^{a}$, Bawar Mohammed Faraj ${ }^{b^{*}}$ and Faraedoon Waly Ahmed ${ }^{c}$ \\ ${ }^{a}$ Department of Mathematics, Harran University, Turkey \\ ${ }^{b}$ Computer Science Department, University of Halabja,Iraq \\ ${ }^{c}$ Department of Physics, University of Halabja,Iraq \\ mmodanli@harran.edu.tr,bawarm.faraj@uoh.edu.iq,faraidun.ahmad@uoh.edu.iq
}

\section{ARTICLE INFO}

Article History:

Received 28 September 2019

Accepted 10 May 2020

Available 01 July 2020

\section{Keywords:}

Time-space telegraph differential equations

Matrix stability

First and second order difference schemes

Approximation solution

AMS Classification 2010:

35-XX; 34K28; 65M12; $74 S 20$

\section{ABSTRACT}

The variable telegraph partial differential equation depend on initial boundary value problem has been studied. The coefficient constant time-space telegraph partial differential equation is obtained from the variable telegraph partial differential equation throughout using Cauchy-Euler formula. The first and second order difference schemes were constructed for both of coefficient constant time-space and variable time-space telegraph partial differential equation. Matrix stability method is used to prove stability of difference schemes for the variable and coefficient telegraph partial differential equation. The variable telegraph partial differential equation and the constant coefficient time-space telegraph partial differential equation are compared with the exact solution. Finally, approximation solution has been found for both equations. The error analysis table presents the obtained numerical results.

\section{$(\mathrm{cc}) \mathrm{EY}$}

\section{Introduction}

Partial differential equations have several applications in engineering, finance, physics and seismology [1-3]. They have several approximation methods which are different from each other. Some of these methods are solvable with respect to variables time and space. The space- heat equations were presented by difference schemes in previous works 446. The partial differential equations depend on time were worked on in some papers [7 9], The telegraph partial differential equations is a special equation of the partial differential equations. In the literature, Telegraph equations can be defined based on time and space. Many important studies have been done on these equations in 10 12. The telegraph partial differential equations were solved by difference schemes and methods in $13-16$.
In this paper, the initial boundary value problem for variable coefficient partial differential equation is investigated

$$
\left\{\begin{array}{l}
\frac{\partial}{\partial t}\left(\alpha(t) u_{t}(t, x)\right)-\frac{\partial}{\partial x}\left(\beta(x) u_{x}(t, x)\right)+p u(t, x) \\
=f(t, x), 0<t<T, 0<x<L \\
u(0, x)=\varphi(x), \quad u_{t}(0, x)=\psi(x), \quad 0 \leq t \leq T, \\
u(t, 0)=g_{1}(t), \quad u(t, L)=g_{2}(t), \quad 0 \leq x \leq L .
\end{array}\right.
$$

Here, $\alpha(t), \beta(x)$ are variable as to $t, x$, respectively. Now, we shall construct first order difference scheme. Then, we will prove the stability estimates for this problem.

\section{First and second order difference schemes for variable telegraph partial differential equation}

If taking as $\alpha(t)=t^{2}, \beta(x)=x^{2}$ and $p=1$ in the formula (1), this formula can be written as follow

*Corresponding Author 


$$
\left\{\begin{array}{l}
t^{2} u_{t t}(t, x)+2 t u_{t}(t, x)-x^{2} u_{x x}(t, x)-2 x u_{x}(t, x) \\
+u(t, x)=f(t, x), 1<t<e^{T}, 1<x<e^{L} \\
u(0, x)=\operatorname{In}(\varphi(x)), u_{t}(0, x)=\operatorname{In}(\psi(x)) \\
u(t, 0)=u(t, L)=0, \quad 1 \leq t \leq e^{T}, 1 \leq x \leq e^{L}
\end{array}\right.
$$

This equation represents a variable time-space telegraph partial differential equation. It is not easy to find out the analytical solution of this equation.

Therefore, if the Cauchy-Euler formula is applied to the last part of the equation separately for the $x$ and $t$ variables, the formula (2) can be written as

$$
\left\{\begin{array}{l}
u_{t t}(t, x)+u_{t}(t, x)-u_{x x}(t, x)-u_{x}(t, x)+u(t, x) \\
=f(t, x), 0<t<T, 0<x<L \\
u(0, x)=\varphi(x), \quad u_{t}(0, x)=\psi(x), \quad 0 \leq t \leq T \\
u(t, 0)=u(t, L)=0, \quad 0 \leq x \leq L .
\end{array}\right.
$$

The problem (3) is a coefficient time-space telegraph partial differential equation.

Now, we shall construct the first and the second order of accuracy difference scheme for the equation (2). In the first step, we consider the set $w_{\tau, h}=[0,1]_{\tau} \times[0, \pi]_{h}$ of a family of grid points depending on the small parameters $\tau$ and $h$. To evaluate difference scheme for problem (2), the following formula

$[0,1]_{\tau} \times[0, \pi]_{h}=\left\{\left(t_{k}, x_{n}\right): t_{k}=k \tau, 0 \leq k \leq N\right.$,

$\left.N \tau=1, x_{n}=n h, 0 \leq n \leq M ; M h=\pi\right\}$,

is used. For the formula (2), we get the first order difference scheme

$$
\left\{\begin{array}{l}
t_{k}^{2} \frac{u_{n}^{k+1}-2 u_{n}^{k}+u_{n}^{k-1}}{\tau^{2}}+2 t_{k} \frac{u_{n}^{k+1}-u_{n}^{k}}{\tau} \\
-x_{n}^{2} \frac{u_{n+1}^{k}-2 u_{n}^{k}+u_{n-1}^{k}}{h^{2}}-2 x_{n} \frac{u_{n+1}^{k}-u_{n-1}^{k}}{2 h} \\
+u_{n}^{k}=f_{n}^{k}, \quad x_{n}=n h, t_{k}=k \tau, \\
1 \leq k \leq N-1,1 \leq n \leq M-1, \\
u_{0}^{k}=u_{M}^{k}=0, u_{n}^{0}=\operatorname{In}\left(\varphi\left(x_{n}\right)\right), 0 \leq k \leq N \\
\frac{u_{n}^{1}-u_{n}^{0}}{\tau}=\operatorname{In}\left(\psi\left(x_{n}\right)\right), 0 \leq n \leq M,
\end{array}\right.
$$

and the second order difference scheme for the formula (2)

$$
\left\{\begin{array}{l}
t_{k}^{2} \frac{u_{n}^{k+1}-2 u_{n}^{k}+u_{n}^{k-1}}{\tau^{2}}+2 t_{k} \frac{u_{n}^{k+1}-u_{n}^{k-1}}{2 \tau} \\
-\frac{x_{n}^{2}}{2} \frac{u_{n+1}^{k+1}-2 u_{n}^{k+1}+u_{n-1}^{k+1}}{h^{2}} \\
-\frac{x_{n}^{2}}{2} \frac{u_{n+1}^{k-1}-2 u_{n}^{k-1}+u_{n-1}^{k-1}}{h^{2}} \\
-\frac{x_{n}}{2} \frac{u_{n+1}^{k+1}-u_{n-1}^{k+1}}{h}-\frac{x_{n}}{2} \frac{u_{n+1}^{k-1}-u_{n-1}^{k-1}}{h} \\
+\frac{1}{2} u_{n}^{k+1}+\frac{1}{2} u_{n}^{k-1}=f_{n}^{k}, \\
x_{n}=n h, t_{k}=k \tau, 1 \leq k \leq N-1,1 \leq n \leq M-1, \\
\frac{u_{n}^{1}-u_{n}^{0}}{\tau}=\operatorname{In}\left(\psi\left(x_{n}\right)\right)+\frac{\tau}{2} \frac{u_{n}^{2}-2 u_{n}^{1}+u_{n}^{0}}{\tau^{2}}, \\
u_{n}^{0}=\operatorname{In}\left(\varphi\left(x_{n}\right)\right), u_{0}^{k}=u_{M}^{k}=0, \\
0 \leq k \leq N, 0 \leq n \leq M .
\end{array}\right.
$$

Similarly, the first order difference schemes for the formula (3) are

$$
\left\{\begin{array}{l}
\frac{u_{n}^{k+1}-2 u_{n}^{k}+u_{n}^{k-1}}{\tau^{2}}+\frac{u_{n}^{k+1}-u_{n}^{k}}{\tau}-\frac{u_{n+1}^{k}-2 u_{n}^{k}+u_{n-1}^{k}}{h^{2}} \\
-\frac{u_{n+1}^{k}-u_{n-1}^{k}}{2 h}+u_{n}^{k}=f_{n}^{k}, \quad x_{n}=n h, t_{k}=k \tau, \\
1 \leq k \leq N-1,1 \leq n \leq M-1, \\
u_{0}^{k}=u_{M}^{k}=0, u_{n}^{0}=\varphi\left(x_{n}\right), \frac{u_{n}^{1}-u_{n}^{0}}{\tau}=\psi\left(x_{n}\right), \\
0 \leq k \leq N, 0 \leq n \leq M,
\end{array}\right.
$$

and the second order difference schemes

$$
\left\{\begin{array}{l}
\frac{u_{n}^{k+1}-2 u_{n}^{k}+u_{n}^{k-1}}{\tau^{2}}+\frac{u_{n}^{k+1}-u_{n}^{k-1}}{2 \tau} \\
-\frac{1}{2} \frac{u_{n+1}^{k+1}-2 u_{n}^{k+1}+u_{n-1}^{k+1}}{h^{2}} \\
-\frac{1}{2} \frac{u_{n+1}^{k-1}-2 u_{n}^{k-1}+u_{n-1}^{k-1}}{h^{2}} \\
-\frac{1}{4} \frac{u_{n+1}^{k+1}-u_{n-1}^{k+1}}{h}-\frac{1}{4} \frac{u_{n+1}^{k-1}-u_{n-1}^{k-1}}{h} \\
+\frac{1}{2} u_{n}^{k+1}+\frac{1}{2} u_{n}^{k-1}=f_{n}^{k}, \\
x_{n}=n h, t_{k}=k \tau, 1 \leq k \leq N-1,1 \leq n \leq M-1, \\
u_{n}^{0}=\varphi(x), \frac{u_{n}^{1}-u_{n}^{0}}{\tau}=\psi(x)+\frac{\tau}{2} \frac{u_{n}^{2}-2 u_{n}^{1}+u_{n}^{0}}{\tau^{2}}, \\
u_{0}^{k}=u_{M}^{k}=0, \quad 0 \leq k \leq N, 0 \leq n \leq M .
\end{array}\right.
$$

The formula (4) is rewritten as 


$$
\begin{aligned}
& \left(\frac{t_{k}^{2}}{\tau^{2}}+2 \frac{t_{k}}{\tau}\right) u_{n}^{k+1}+\left(-\frac{x_{n}^{2}}{h^{2}}-\frac{x_{k}}{h}\right) u_{n+1}^{k} \\
& +\left(-2 \frac{t_{k}^{2}}{\tau^{2}}-2 \frac{t_{k}}{\tau}+1+2 \frac{x_{n}^{2}}{h^{2}}\right) u_{n}^{k} \\
& +\left(-\frac{x_{n}^{2}}{h^{2}}+\frac{x_{n}}{h}\right) u_{n-1}^{k}+\left(\frac{t_{k}^{2}}{\tau^{2}}\right) u_{n}^{k-1}=f_{n}^{k}
\end{aligned}
$$

Then, the last formula can be written as

$$
a u_{n}^{k+1}+b u_{n+1}^{k}+c u_{n}^{k}+d u_{n-1}^{k}+e u_{n}^{k-1}=f_{n}^{k} .
$$

Here,

$a=\frac{t_{k}^{2}}{\tau^{2}}+2 \frac{t_{k}}{\tau}, b=-\frac{x_{n}^{2}}{h^{2}}-\frac{x_{k}}{h}$,

$c=-2 \frac{t_{k}^{2}}{\tau^{2}}-2 \frac{t_{k}}{\tau}+1+2 \frac{x_{n}^{2}}{h^{2}}$

$d=-\frac{x_{n}^{2}}{h^{2}}+\frac{x_{n}}{h}$ and $e=\frac{t_{k}^{2}}{\tau^{2}}$.

From the formula (9), the following matrices' formulas are obtained as

$$
A U^{k+1}+B U^{k}+C U^{k-1}=\phi^{k}
$$

where, $A, B$ and $C$ are $(N+1) \times(N+1)$ matrix, $U^{k+1}, U^{k}, U^{k-1}$ and $\phi^{k}=F_{n}^{k}$ is $(N+1) \times 1$ vector as the following

$$
A=a\left[\begin{array}{ccccccc}
0 & 0 & 0 & \ldots & 0 & 0 & 0 \\
0 & 1 & 0 & \ldots & 0 & 0 & 0 \\
0 & 0 & 1 & \ldots & 0 & 0 & 0 \\
\vdots & \vdots & \vdots & \ddots & \vdots & \vdots & \vdots \\
0 & 0 & 0 & \ldots & 1 & 0 & 0 \\
0 & 0 & 0 & \ldots & 0 & 1 & 0 \\
0 & 0 & 0 & \ldots & 0 & 0 & 0
\end{array}\right]_{(N+1) \times(N+1)}
$$

$$
B=\left[\begin{array}{ccccccccc}
c & b & 0 & 0 & \ldots & 0 & 0 & 0 & 0 \\
d & c & b & 0 & \ldots & 0 & 0 & 0 & 0 \\
0 & d & c & b & \ldots & 0 & 0 & 0 & 0 \\
0 & 0 & d & c & \ldots & 0 & 0 & 0 & 0 \\
\vdots & \vdots & \vdots & \vdots & \ddots & \vdots & \vdots & \vdots & \vdots \\
0 & 0 & 0 & 0 & \ldots & c & b & 0 & 0 \\
0 & 0 & 0 & 0 & \ldots & d & c & b & 0 \\
0 & 0 & 0 & 0 & \ldots & 0 & d & c & b \\
0 & 0 & 0 & 0 & \ldots & 0 & 0 & d & c
\end{array}\right]_{(N+1) \times(N+1)}
$$

$$
C=e\left[\begin{array}{ccccccc}
1 & 0 & 0 & \ldots & 0 & 0 & 0 \\
0 & 1 & 0 & \ldots & 0 & 0 & 0 \\
0 & 0 & 1 & \ldots & 0 & 0 & 0 \\
\vdots & \vdots & \vdots & \ddots & \vdots & \vdots & \vdots \\
0 & 0 & 0 & \ldots & 1 & 0 & 0 \\
0 & 0 & 0 & \ldots & 0 & 1 & 0 \\
0 & 0 & 0 & \ldots & 0 & 0 & 1
\end{array}\right]_{(N+1) \times(N+1)}
$$

$$
\begin{aligned}
U^{k-1}=\left[\begin{array}{c}
u_{0}^{k-1} \\
u_{1}^{k-1} \\
u_{2}^{k-1} \\
\vdots \\
u_{N-1}^{k-1} \\
u_{N}^{k-1}
\end{array}\right]_{(N+1) \times 1} U^{k}=\left[\begin{array}{c}
u_{0}^{k} \\
u_{1}^{k} \\
u_{2}^{k} \\
\vdots \\
u_{N-1}^{k} \\
u_{N}^{k}
\end{array}\right]_{(N+1) \times 1} \\
U^{k+1}=\left[\begin{array}{c}
u_{0}^{k+1} \\
u_{1}^{k+1} \\
u_{2}^{k+1} \\
\vdots \\
u_{N-1}^{k+1} \\
u_{N}^{k+1}
\end{array}\right]_{(N+1) \times 1 .}
\end{aligned}
$$

Modified Gauss elimination method is applied to solve the above difference equations. After that, a solution of the matrix equation is looked for as the following form

$u_{j}=\alpha_{j+1} u_{j+1}+\beta_{j+1} ; u_{M}=0 ; j=M-1, \ldots, 2,1$.

Using boundary conditions, the formula

$$
u_{0}=\alpha_{1} u_{1}+\beta_{1}=0
$$

is obtained. Then, $\alpha_{1}$ is obtained the $(N+$ $1) \times(N+1)$ zero matrix and $\beta_{1}$ is obtained the $(N+1) \times 1$ zero column vector. Using the formula (14), the following formula is found

$$
\begin{aligned}
A u_{j+1}+B\left[\alpha_{j+1} u_{j+1}+\beta_{j+1}\right] & +C\left[\alpha_{j} u_{j}+\beta_{j}\right]=\phi_{j}, \\
A u_{j+1}+B\left[\alpha_{j+1} u_{j+1}+\beta_{j+1}\right] & +C\left[\alpha _ { j } \left[\alpha_{j+1} u_{j+1}\right.\right. \\
& \left.\left.+\beta_{j+1}\right]+\beta_{j}\right]=\phi_{j}, \\
A u_{j+1}+B \alpha_{j+1} u_{j+1}+B \beta_{j+1} & +C \alpha_{j} \alpha_{j+1} u_{j+1}
\end{aligned}
$$$$
+C \alpha_{j} \beta_{j+1}+C \beta_{j}=\phi_{j},
$$ 


$$
\begin{gathered}
{\left[A+B \alpha_{j+1}+C \alpha_{j} \alpha_{j+1}\right] u_{j+1}+B \beta_{j+1}} \\
+C \alpha_{j} \beta_{j+1}+C \beta_{j}=\phi_{j}
\end{gathered}
$$

and then also

$$
\begin{aligned}
& {\left[A+B \alpha_{j+1}+C \alpha_{j} \alpha_{j+1}\right] u_{j+1}=0} \\
& \text { and } \\
& B \beta_{j+1}+C \alpha_{j} \beta_{j+1}+C \beta_{j}=\phi_{j} .
\end{aligned}
$$

From the (15), the formulas are found

$$
\alpha_{j+1}=-\left(B+C \alpha_{j}\right)^{-1} A
$$

and

$$
\begin{aligned}
& \text { and } \\
& \beta_{j+1}=\left(B+C \alpha_{j}\right)^{-1}\left(D \phi-C \beta_{j}\right), \quad j=1,2, \ldots, M-1 .
\end{aligned}=\frac{1-2 \frac{t_{k}^{2}}{\tau^{2}}-2 \frac{t_{k}}{\tau}}{\frac{t_{k}^{2}}{\tau^{2}}+\frac{t_{k}}{\tau}}
$$

Here, $\alpha_{j}$ is $(N+1) \times(N+1)$ zero matrix and $\beta_{j}$ is $(N+1) \times 1$ zero column vector.

Now, we shall prove the stability estimate by applying the method of analyzing the eigenvalues of the iteration matrices of the schemes for the formula (44). For this, we express $\|A\|=\|A\|_{\infty}=\max _{1 \leq k \leq N-1}\left[\sum_{i=1}^{N-1}\left|a_{k m}\right|\right]$, where $A=\left[a_{k m}\right]_{(N-1) \times(N-1)}, I$ is unit matrix.

Let $\rho(A)$ be the spectral radius of a matrix $A$, which means the maximum of the absolute value of the eigenvalues of the matrix $A$. We can write the following theorem.

Theorem 1. If $-2 \frac{t_{k}^{2}}{\tau^{2}}-2 \frac{t_{k}}{\tau}+1+2 \frac{x_{n}^{2}}{h^{2}}>0$, then, the difference scheme (4) is stable.

Proof. From the method [18, we should prove that $\rho\left(\alpha_{n}\right)<1,1 \leq n \leq M$.

$\rho\left(\alpha_{1}\right)=0<1$ is clearly.

$$
\begin{aligned}
& \rho\left(\alpha_{2}\right)=\left\|-B A^{-1}\right\| \leq\|-B\|\left\|A^{-1}\right\| \\
& =\|B\| \frac{1}{\min _{1 \leq k \leq N-1}\left\{\left|a_{k k}\right|-\sum_{\substack{m \neq k, m=1}}^{N-1}\left|a_{k m}\right|\right\}}
\end{aligned}
$$

$=\frac{\left|-2 \frac{t_{k}^{2}}{\tau^{2}}-2 \frac{t_{k}}{\tau}+1+2 \frac{x_{n}^{2}}{h^{2}}\right|}{\left|\frac{t_{k}^{2}}{\tau^{2}}+\frac{t_{k}}{\tau}\right|}$

$+\frac{\left|-\frac{x_{n}^{2}}{h^{2}}-\frac{x_{k}}{h}\right|+\left|-\frac{x_{n}^{2}}{h^{2}}+\frac{x_{k}}{h}\right|}{\left|\frac{t_{k}^{2}}{\tau^{2}}+\frac{t_{k}}{\tau}\right|}$

$=\frac{-2 \frac{t_{k}^{2}}{\tau^{2}}-2 \frac{t_{k}}{\tau}+1+2 \frac{x_{n}^{2}}{h^{2}}-\frac{x_{n}^{2}}{h^{2}}-\frac{x_{k}}{h}-\frac{x_{n}^{2}}{h^{2}}+\frac{x_{k}}{h}}{\frac{t_{k}^{2}}{\tau^{2}}+\frac{t_{k}}{\tau}}$ $=\frac{1-2\left(k^{2}+k\right)}{\left(k^{2}+k\right)} \leq 1, k=1,2, \ldots M$.

If $\rho\left(\alpha_{n}\right)<1$, let us calculate $\rho\left(\alpha_{n+1}\right)$ for the formula (3) and procedure [19]. We know that $\alpha_{n i}=\rho\left(\alpha_{n}\right)$ and $0 \leq \rho\left(\alpha_{n}\right)<1$ for $2 \leq i \leq N+1$. Then, we can obtain that $\rho\left(\alpha_{n+1}\right)<1$. Thus, the proof of the theorem is completed.

For the stability estimate of the second order difference schemes formula (5), a similar procedure can be used. The stability estimates of the formulas (6) and (7) were given in the [13, [17].

Now let's find the approximate solutions of a few examples for the application of these theoretical expressions.

\section{Numerical experiments}

In this section, some numerical example for the telegraph partial differential equation by the first and second order difference schemes method will be present. We can calculate the maximum norm of the error of the numerical solution as

$$
E_{M}^{N}=\max _{1 \leq k \leq N-1,1 \leq n \leq M-1}\left|u\left(t_{k}, x_{n}\right)-u_{n}^{k}\right| .
$$

Where $u\left(t_{k}, x_{n}\right)$ represents the exact solution and $u_{n}^{k}$ represents numerical solution at points $\left(t_{k}, x_{n}\right)$. Result of calculations tell us the second order has more accurate than the first order of accuracy difference scheme.

Example 1. Consider the following initial boundary value problem for Telegraph partial differential equation 
$\left\{\begin{array}{l}u_{t t}(t, x)+u_{t}(t, x)-u_{x x}(t, x)-u_{x}(t, x)+u(t, x) \\ =\cos (x-t)-\sin (x) \cos (t), 0<t<1, \quad 0<x<\pi \\ u(0, x)=-\sin (x), \quad u_{t}(0, x)=0 \\ u(t, 0)=u(t, \pi)=0,0 \leq t \leq 1,0 \leq x \leq \pi\end{array}\right.$

Using the Laplace transform method, the exact solution of the problem (16) is $u(x, t)=$ $-\sin (x) \cos (t)$. Error analysis Table 1 is shown the approximation solution of the problem (16).

Table 1. Error analysis for exact and approximation solution for example [16.

\begin{tabular}{ccc}
\hline $\begin{array}{c}\tau=1 / N, \\
h=\pi / M\end{array}$ & $\begin{array}{c}\text { First } \\
\text { Order } \\
\text { Difference } \\
\text { Scheme }\end{array}$ & $\begin{array}{c}\text { Second } \\
\text { Order } \\
\text { Difference } \\
\text { Scheme }\end{array}$ \\
\hline$N=M=20$ & $1.1102 \times 10^{-2}$ & $1.8527 \times 10^{-3}$ \\
\hline$N=M=50$ & $3.8794 \times 10^{-3}$ & $2.9979 \times 10^{-4}$ \\
\hline$N=M=100$ & $1.8400 \times 10^{-3}$ & $7.5204 \times 10^{-5}$ \\
\hline$N=M=200$ & $8.9448 \times 10^{-4}$ & $1.8815 \times 10^{-5}$ \\
\hline$N=M=400$ & $4.4078 \times 10^{-4}$ & $4.7025 \times 10^{-6}$ \\
\hline$N=M=600$ & $2.9241 \times 10^{-4}$ & $2.0896 \times 10^{-6}$ \\
\hline
\end{tabular}

Example 2. Investigate the following initial boundary value problem for Telegraph partial differential equation

$$
\left\{\begin{array}{l}
u_{t t}(t, x)+u_{t}(t, x)-u_{x x}(t, x)-u_{x}(t, x)+u(t, x) \\
=\left(x^{2}-2 x-2\right) e^{-t}+\pi(1-x) e^{-t}, \\
0<t<1,0<x<\pi, \\
u(0, x)=x(x-\pi), \quad u_{t}(0, x)=-x(x-\pi), \\
u(t, 0)=u(t, \pi)=0, \quad 0 \leq t \leq 1,0 \leq x \leq \pi .
\end{array}\right.
$$

The exact solution of the problem (17) is $u(x, t)=$ $\left(x^{2}-\pi x\right) e^{-t}$. Error analysis Table 2 is shown the approximation solution of the problem (17).
Table 2. Error analysis for exact and approximation solution for example 17.

\begin{tabular}{|c|c|c|}
\hline $\begin{array}{c}\tau=1 / N \\
h=\pi / M\end{array}$ & $\begin{array}{c}\text { First } \\
\text { Order } \\
\text { Difference } \\
\text { Scheme }\end{array}$ & $\begin{array}{c}\text { Second } \\
\text { Order } \\
\text { Difference } \\
\text { Scheme }\end{array}$ \\
\hline$N=M=20$ & $3.7052 \times 10^{-2}$ & $2.1852 \times 10^{-3}$ \\
\hline$N=M=50$ & $1.5780 \times 10^{-2}$ & $3.5362 \times 10^{-4}$ \\
\hline$N=M=100$ & $8.0644 \times 10^{-3}$ & $8.8693 \times 10^{-5}$ \\
\hline$N=M=200$ & $4.0783 \times 10^{-3}$ & $2.2207 \times 10^{-5}$ \\
\hline$N=M=400$ & $2.0505 \times 10^{-3}$ & $5.5558 \times 10^{-6}$ \\
\hline$N=M=600$ & $1.3695 \times 10^{-3}$ & $2.4698 \times 10^{-6}$ \\
\hline
\end{tabular}

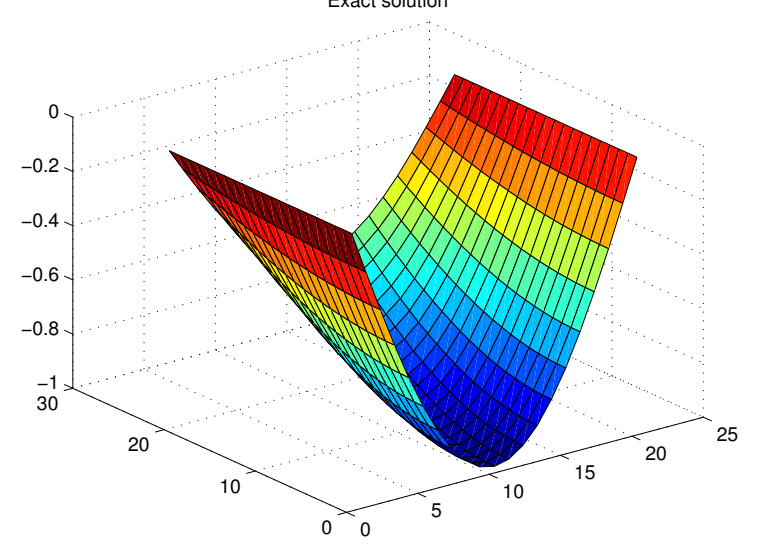

Figure 1. Figure of exact solution for problen[16], where $\mathrm{N}=\mathrm{M}=20$.

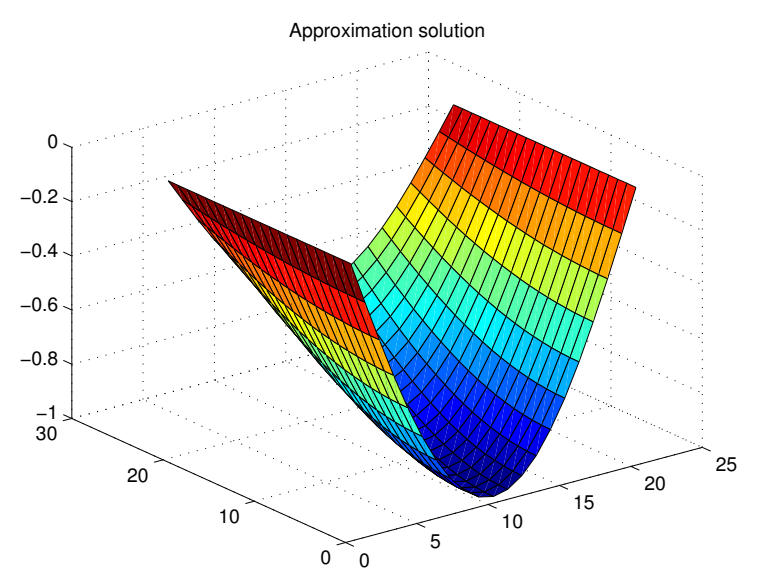

Figure 2. Figure of approximation solution for problem [16, where $\mathrm{N}=\mathrm{M}=20$. 
Remark 1. Using the first order difference scheme formula (4), we obtain the the following numerical results for the problem (2) and example (17). For example; Taking $N=21, M=20$, we obtain maxerror $=8.7021 \times 10^{-1}$. For these values, the figures are the added as follow:

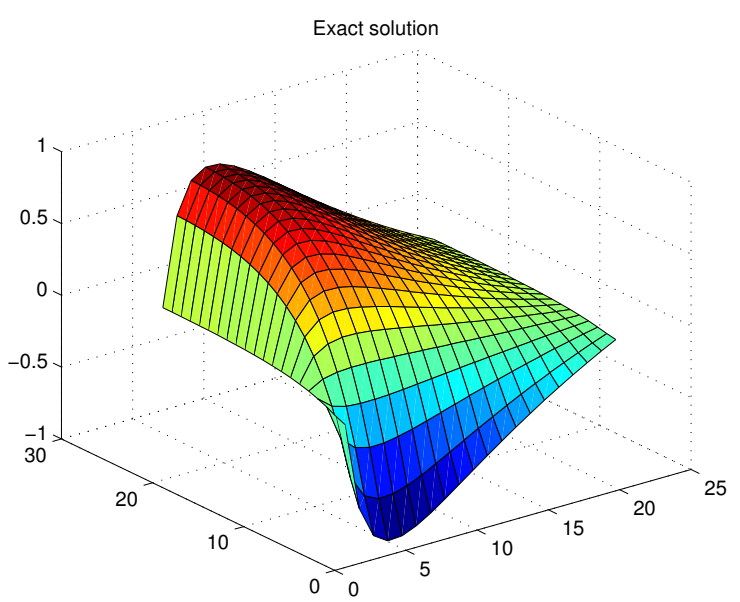

Figure 3. Figure of exact solution for problem(2) and example (16), where $\mathrm{N}=21, \mathrm{M}=20$.

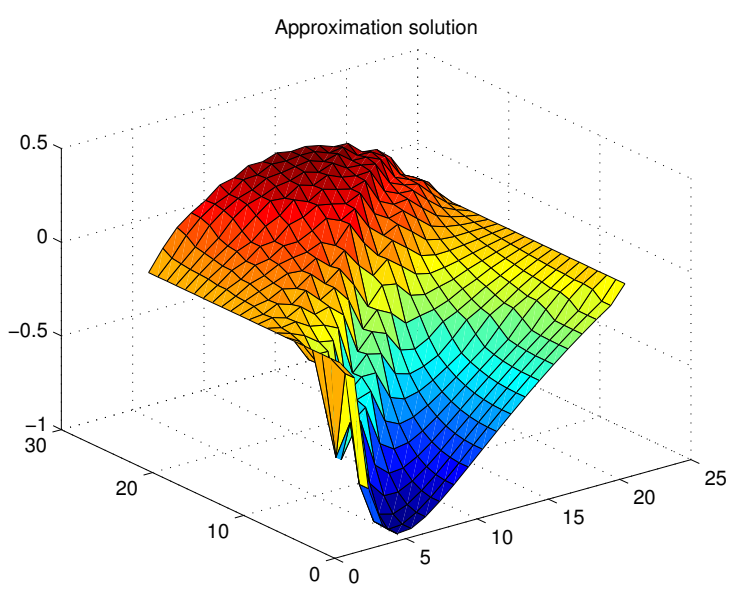

Figure 4. Figure of approximation solution for problem(2) and example (16), where $\mathrm{N}=21, \mathrm{M}=20$.

Remark 2. The following results are obtained through using the Cauchy-Euler formula:

$i$. The non-uniform region becomes a smooth region. And this is easier made calculation of the Matlab program.

ii. This also provides to obtain more appropriate and beautiful numerical results.

\section{Conclusion}

In this paper, the variable telegraph partial differential equation has been investigated. Then, this equation is transformed to the constant coefficient via using Cauchy-Euler formula. For this equation, we construct the first and second order difference schemes. Stability estimate is proved for these difference schemes. The exact and approximate solution of the problem were compared to obtain the error analysis in the maximum norm. Numerical examples show that this method is appropriate for this problem.

\section{Acknowledgments}

We would like to thank the referees for their valuable comments and suggestions to improve our paper.

\section{References}

[1] Çelik, C., \& Duman, M.(2012). CrankNicolson method for the fractional diffusion equation with the Riesz fractional derivative. Journal of computational physics, 231(4), 1743-1750.

[2] Gorial, I. I. (2011). Numerical methods for fractional reaction-dispersion equation with Riesz space fractional derivative. Engineering and Technology Journal, 29(4), 709-715.

[3] Jafari, H., \& Daftardar-Gejji, V. (2006). Solving linear and nonlinear fractional diffusion and wave equations by Adomian decomposition. Applied Mathematics and Computation, 180(2), 488-497.

[4] Karatay, I., Bayramoglu, ŞR., \& Şahin, A. (2011). Implicit difference approximation for the time fractional heat equation with the nonlocal condition. Applied Numerical Mathematics, 61(12), 1281-1288.

[5] Su, L., Wang, W., \& Yang, Z. (2009). Finite difference approximations for the fractional advection-diffusion equation. Physics Letters A, 373(48), 4405-4408.

[6] Tadjeran, C., Meerschaert, M. M., Scheffler, H.P.(2006). A second order accurate numerical approximation for the fractional diffusion equation. Journal of computational physics, 213(1), 205-213.

[7] Nouy, A. (2010). A priori model reduction through proper generalized decomposition for solving time-dependent partial differential equations. Computer Methods in Applied Mechanics and Engineering, 199(23-24), 1603-1626.

[8] Wu, J. (1996). Theory and applications of partial functional differential equations, Springer-Verlag, New York.

[9] Pontryagin, L. S. (2018). Mathematical theory of optimal processes, Routledge, London. 
[10] He, J. H. (2008). Recent development of the homotopy perturbation method. Topological methods in nonlinear analysis, 31(2), 205-209.

[11] Holmes, E. E., Lewis, M. A., Banks, J. E., \& Veit, R. R. (1994). Partial differential equations in ecology: spatial interactions and population dynamics. Ecology, 75(1), 17-29.

[12] Dehghan, M., Shokri, A. (2008). A numerical method for solving the hyperbolic telegraph equation. Numerical Methods for Partial Differential Equations, 24(4), 1080-1093.

[13] Faraj, B., \& Modanli, M. (2017). Using difference scheme method for the numerical solution of telegraph partial differential equation. Journal of Garmian University, 3, 157-163.

[14] Ashyralyev, A., \& Modanli, M. (2015). An operator method for telegraph partial differential and difference equations. Boundary Value Problems, 41(1), 1-17.

[15] Ashyralyev, A., \& Modanli, M. (2015). Nonlocal boundary value problem for telegraph equations. AIP Conference Proceedings, 1676(1), 020078-1 - 020078-4.

[16] Ashyralyev A, \& Modanli, M. (2014). A numerical solution for a telegraph equation. AIP Conference Proceedings, 1611(1), 300-304.
[17] Faraj, B. M. (2018). Difference scheme methods for telegraph partial differential equations. MSc Thesis. Harran University.

[18] Smith, G. D. (1985). Numerical solution of partial differential equations: finite difference methods. Oxford university press.

[19] Richtmyer, R. D., \& Morton, K. W. (1994). Difference methods for initial-value problems, Krieger Publishing Co., 2nd ed., Portland, USA.

Mahmut Modanli is an associate professor in applied mathematics holding PhD at Department of Mathematics, Faculty of Arts and Sciences, Harran University, Sanliurfa, 63010, Turkey.

(1) http://orcid.org/0000-0002-7743-3512

Bawar Mohammed Faraj is an assistant lecturer holding Msc in applied mathematics at Computer science department, College of Science, University of Halabja, Halabja, 46018, Iraq.

(1) http://orcid.org/0000-0002-7543-2890

Faraedoon Waly Ahmed is an assistant lecturer holding Msc in electrical engineering at Department of Physics, college of Science, University of Halabja, Halabja, 46018, Iraq.

(10) http://orcid.org/0000-0003-3016-6274

An International Journal of Optimization and Control: Theories \& Applications (http://ijocta.balikesir.edu.tr)

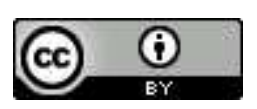

This work is licensed under a Creative Commons Attribution 4.0 International License. The authors retain ownership of the copyright for their article, but they allow anyone to download, reuse, reprint, modify, distribute, and/or copy articles in IJOCTA, so long as the original authors and source are credited. To see the complete license contents, please visit http://creativecommons.org/licenses/by/4.0/. 